\title{
Dual hemispheric processing in a letter matching task
}

\author{
JEFFREY CONEY \\ Murdoch University, Murdoch, Western Australia, Australia
}

\begin{abstract}
It has been reported that performance on recognition, detection, and matching tasks is enhanced if stimuli are projected to both sides of the visual field rather than to one side alone (Dimond, 1972). The present study investigated the claim that this phenomenon is due to the distribution of the burden of perceptual processing between the hemispheres. Three experiments were carried out using a matching paradigm in which RT and response errors were recorded. In all experiments, subjects were required to match two letters that were displayed separately on either side of a central fixation point (bilateral presentation) or were displayed together on the same side of the visual field (unilateral presentation). It was found that although lateral interference between adjacent stimuli was significantly implicated in the phenomenon, a strong residual effect, which could be tentatively ascribed to hemispheric mechanisms, remained in relation to letter name matches. It is argued that a model based on parallel hemispheric decision processes provides a better account of the data than does one based upon the notion of distributed perceptual processing.
\end{abstract}

Several studies (e.g., Dimond, 1970, 1972; Elithorn $\&$ Barnett, 1967) have shown that performance on a reaction time (RT) task may be facilitated by projecting component stimuli of the task to different, rather than same, sides of the visual field. Dimond (1970), for example, presented arrow shapes to the left, right, or both sides of the visual field. Subjects were instructed to respond with the hand indicated by the arrow. On some trials, two arrows were presented simultaneously, one pointing in each direction, and a double response was required. On these trials, the two arrows were presented in either the left or the right visual field, or were divided so that one arrow appeared on each side of the visual field. The results of this experiment were striking. When a double response was required, RT was some $95 \mathrm{msec}$ shorter when the arrows were divided between the two sides of the visual field. Dimond attributed this finding to the distribution of the burden of perceptual processing between the cerebral hemispheres. He argued that the arrival of stimuli in separate hemispheres, via opposite sides of the visual field, permits simultaneous analysis and encoding to occur in the two perceptual systems, thus reducing the overall time ordinarily required for such operations. In commenting on this explanation, he noted that if each hemisphere shared stimulus information with the other immediately upon receiving it, one would not expect the projection of stimuli to same or separate visual fields to have any differential effect upon performance.

This implies that when stimuli are projected to both sides of the visual field, the two hemispheres are able to

The author wishes to acknowledge the advice and support provided by Kim Kirsner during the execution of this study. The author's mailing address is: Department of Psychology, Murdoch University, Murdoch, W.A. 6150 , Australia. process their respective stimuli in parallel. However, since responses to double signals were considerably prolonged over responses to single signals, it is equally clear that the two hemispheric systems are not able to function with a sufficient degree of independence to permit two stimuli to be fully processed in the time that it takes to process one. Dimond concluded, therefore, that some initial processing occurs on an independent basis in each hemisphere, but that this is followed by interhemispheric communication for the purpose of integration of subsequent activities.

The general explanation offered by Dimond in relation to this phenomenon appears to be quite reasonable. In view of the existence of anatomically distinct visual systems in the occipital cortex of each hemisphere, it seems likely that separately received stimuli should enjoy some degree of independent processing. That this should result in faster or more efficient performance is similarly unsurprising. These observations, however, apply to experimental tasks constructed in such a way that the information presented to each hemisphere can be processed without the necessity for interhemispheric coordination or cross-reference. A study by Davis and Schmit (1973) is therefore of especial interest, since it apparently demonstrates a substantial advantage for distributed input in the context of an experimental task that cannot be performed without interhemispheric communication of stimulus information. This study required subjects to match two letters presented either to the same or to different sides of the visual field (Posner \& Mitchell, 1967). One group of subjects was instructed to match stimulus letters on the basis of their physical similarity; a second group was instructed to match on the basis of names. Under both conditions, it was found that projection of the members of each stimulus pair to separate sides of the visual field 
resulted in significantly shorter latencies than projection of both members to the same side of the visual field.

Davis and Schmit related their results to Dimond's work, and sought to explain their findings along similar lines by arguing that the projection of stimuli to separate hemispheres resulted in a reduction in overall processing time by allowing analysis of the stimuli to proceed in parallel. The results of this study are nonetheless somewhat surprising. In order for a comparison to occur between stimuli presented to different cerebral hemispheres, it is logically necessary for some stimulus information to be transmitted between the hemispheres, thus raising the possibility of a delay relative to the situation in which both stimuli arrive in the same hemisphere. Although interhemispheric transmission time may not be a significant factor in this regard (Bradshaw \& Nettleton, 1983; Swanson, Ledlow, \& Kinsbourne, 1978), there is evidence to suggest that each hemisphere has idiosyncratic ways of encoding similar stimulus information (e.g., Gazzaniga \& Ledoux, 1978; Kinsbourne, 1978; Moscovitch, 1979). It is at least arguable, therefore, that stimuli encoded in one hemisphere might be relatively difficult to match against stimuli encoded in the other hemisphere, whereas stimuli encoded within the same hemisphere might be matched with comparative ease. In view of these considerations, the relatively large effects reported by Davis and Schmit raise the question of whether the advantage of bilaterally distributing stimuli can really be attributed to parallel processing by the cerebral hemispheres. As these studies were explicitly directed to the investigation of laterality effects, and thus incorporated the procedures traditionally employed to ensure the selective delivery of information to designated hemispheres, it would seem natural to interpret the results in terms of hemispheric processes. It is important to recognize, however, that this interpretation remains essentially speculative (Colbourn, 1978 , and White, 1973, make a similar point in relation to the interpretation of visual asymmetry studies). The hemispheric hypothesis remains essentially untested. It is relatively easy, furthermore, to conceive of alternative explanations of the bilateral distribution effect which do not invoke hemispheric mechanisms. Such alternatives do not appear to have been given adequate consideration.

Even if a general hemispheric explanation were to be accepted in principle, there remains the problem of elucidating the actual information processing mechanisms underlying the bilateral distribution effect. Dimond has proposed the perceptual processing stage as the locus of the effect, a view endorsed by Davis and Schmit. Once again, however, it must be observed that this notion has little more than theoretical appeal in its favor. No effort has been made to provide the necessary empirical support for this view, and equally little consideration appears to have been given to the possible involvement of later stages of processing in the bilateral distribution effect. The present study was thus conceived as an attempt to (1) investigate alternative explanations of the bilateral distri- bution effect, (2) determine whether some more direct evidence could be obtained in respect of the hemispheric hypothesis, and (3) elucidate the information processing mechanisms involved in the effect.

\section{EXPERIMENT 1}

A basic aim of Experiment 1 was to determine whether the effects reported by Davis and Schmit could be successfully replicated. In particular, it was considered desirable to investigate the relationship between physical and name matches and the effect of the bilateral distribution. Davis and Schmit reported that physical and name matches were equally facilitated under bilateral distribution of letter pairs. However, this finding is difficult to reconcile with the evidence that qualitatively different processes underlie these two types of matches (see, e.g., Posner, 1970; Posner, Boies, Eichelman, \& Taylor, 1969). As observed earlier, Davis and Schmit have followed Dimond in arguing that the advantage conferred by distributing stimulus pairs across the visual field derives from the sharing of the burden of perceptual analysis between the hemispheres. This implies that tasks of different duration should enjoy a proportionate, rather than fixed, reduction in processing time. In the present context, the absolute value of the reduction will depend upon the duration of perceptual processes. If, as seems likely, the fact that name matches invariably require more time than physical matches is reflected in the relative durations of the corresponding perceptual processes, then bilateral distribution might be expected to produce a greater absolute saving in the time required for the development of name codes, as compared with the saving for physical codes. In fact, as indicated earlier, Davis and Schmit found no significant difference between the effect of bilateral distribution on physical and name matches. However, the fact that different groups of subjects were assigned to physical and name match conditions in Davis and Schmit's study raises the possibility that their experiment may not have been sensitive enough to reveal the relationship between match type and bilateral distribution (Winer, 1971). It was intended to investigate this possibility by incorporating match type as a within-subjects variable in $\mathrm{Ex}$ periment 1.

A further aim of the present experiment concerned the role that nonhemispheric mechanisms might play in producing the bilateral distribution effect. A consideration of Davis and Schmit's experiment suggests one such mechanism. When presenting a pair of letters unilaterally, Davis and Schmit displayed the letters in vertical alignment (i.e., one above the other). In contrast to this, letters presented bilaterally were aligned horizontally. Research into the effect of Western left-right reading habits on the perception of such displays (e.g., Heron, 1957; White, 1969) suggests that in this experiment letter pairs presented unilaterally may have been perceived less easily than letter pairs presented bilaterally. It was decided to 
investigate this possibility in Experiment 1 by comparing the relative effects of vertical and horizontal pair alignment in unilateral displays.

\section{Method}

Subjects. The subjects consisted of 22 undergraduate students enrolled in an introductory psychology course. All subjects were required to have good unaided vision for material presented at a distance of $45 \mathrm{~cm}$, and all were right-handed by self-report. A different sample of subjects was selected for each of the three experiments in the present study, and the above selection criteria were applied to each sample. The present sample comprised 11 males and 11 females, varying in age from 17 to 21 years. The subjects were paid at standard rates for their participation in the experiment.

Apparatus. All experiments in the present study were carried out with the aid of a DIGITAL PDP-11/10 processor, which included a line-time clock and a programmable real-time clock for precise measurement and control of experimental events. A custombuilt hardware random-number generator was interfaced to the computer to provide true random-number sequences where required in the experiment.

Subjects were tested in a cubicle isolated from the control room housing the computer. The cubicle contained a BWD 594 oscilloscope on which stimulus material was presented. Stimuli were plotted under computer control on a screen with an effective area of $10^{2} \mathrm{~cm}$ and a resolution of $1,000^{2}$ points. The screen featured a P1 phosphor which decays to $10 \%$ of initial intensity in approximately $1 \mathrm{msec}$. A Polaroid filter was fitted to reduce stray reflections. A specially constructed chinrest restricted head movement in relation to the display screen. Instructions and performance feedback generated under computer control were relayed from a TEC video terminal to an OSAKA video monitor in the cubicle. Subject responses were signaled to the computer via a button mounted directly in front of the oscilloscope display screen.

Stimuli. The stimulus ensemble used throughout the present study consisted of the upper- and lowercase representations of the letters $A, E$, and $R$. These letters were selected for their low degree of visual and acoustic similarity, and absence of "analogue" pairing between upper- and lowercase forms (e.g., Cc), It was considered important to employ a stimulus ensemble featuring a very small number of familiar, easily recognized letters in order to ensure that task processing imposed as few demands as possible. It was felt that the possibility of dual hemisphere processing could not reasonably be investigated in the context of a task that made significant demands upon the specialist abilities of one or the other hemisphere.

Photographic transparencies of the letters were produced from Letraset Helvetica Light characters. These transparencies were backprojected onto a large ground-glass screen, enabling the coordinates describing each letter to be determined and entered into a computer disk file. During experimental sessions, the coordinate data were plotted under computer control on the oscilloscope display screen in order to produce the stimulus figures seen by the subject. Prior to the display, each letter was transformed so that, as viewed by the subject, it subtended a visual angle of $1^{\circ}$ along both horizontal and vertical dimensions. This procedure was applied to all letters irrespective of case in order to ensure that letter forms could not be differentiated on the basis of simple size cues.

Procedure. Four factors were manipulated as within-subject variables. On each trial, a subject was briefly presented with a pair of letters for comparison. Match type, which comprised three levels, determined the relationship between the letters to be compared. Pairs of letters could match one another physically (i.e., AA, aa, EE, ee, $R R, r r$ ), match one another in name only (i.e., Aa, Ee, Rr), or be entirely different. Letter pairs in "different" displays were constructed from all combinations of letter and case, with the simple constraint that the letters in each pair be different (e.g., AE, $\mathrm{Ra}$, ea). The transformation of all letter forms to the same physical dimensions irrespective of case eliminated the possibility of subjects' using simple size cues to distinguish between physical matches and name matches or "different" pairs. Stimulus distribution determined the gross spatial relationship between the displayed letters. Unilateral displays presented both letters in the same visual hemifield, whereas bilateral displays contained one letter in each visual hemifield. In unilateral displays alone, visual field determined whether both letters were presented in the left or in the right hemifield, and pair alignment determined whether the adjacent letters were presented in horizontal (i.e., side by side) or vertical (i.e., one above the other) alignment. Match type and stimulus distribution were completely crossed to form six separate conditions; visual field and pair alignment were completely crossed to form four conditions. Since the latter two factors were nested under unilateral conditions, there were a total of 15 separate conditions in all. Data were collected on 360 trials in each session. Match type divided this into 120 trials each for physical matches, name matches, and "different" displays. Stimulus distribution divided the total into 180 unilateral trials and 180 bilateral trials. Unilateral trials were further divided by visual field into 90 right- and 90 left-visual-field trials, and by pair alignment into 90 horizontal and 90 vertical trials.

A subject was seated in the testing cubicle so that his or her eyes were positioned $45 \mathrm{~cm}$ distant from, and directly in front of, the stimulus display screen. A chinrest was provided to ensure that the subject could accurately maintain this position for the duration of the session. Each trial commenced with the appearance of a cross, subtending a visual angle of $0.5^{\circ}$, in the center of the display screen. The subject was instructed to fixate the cross as soon as it appeared. After $600 \mathrm{msec}$, the cross was replaced by a simple dot, which persisted for $400 \mathrm{msec}$ and acted as a final warning signal. The disappearance of the dot was immediately followed by exposure of the stimulus letters for a period of $150 \mathrm{msec}$. In unilateral displays, letter pairs were centered on a point situated at a lateral displacement of $4.0^{\circ}$ of visual angle to the left or right of the fixation point. In the case of both vertical and horizontal pairs, letters were separated by $0.2^{\circ}$ of visual angle. In bilateral displays, a letter was centered on a point $4.0^{\circ}$ to either side of the fixation point. In the course of instruction, the necessity for maintaining central fixation of gaze was strongly emphasized. In view of the fact that "same" judgments were of principal interest at this stage of the investigation, and in order to maximize stability and reliability of response data (Pachella, 1974), the subject was instructed to respond on a GO-NOGO basis (Donders C-reactions). Following stimulus onset, the subject was allowed $2 \mathrm{sec}$ in which to signal a "same" judgment by pressing the response button, or to signal a "different" judgment by withholding a response.

\section{Results}

Figure 1 shows mean RT for all conditions in Experiment 1 except those pertaining to visual field. A level of significance of $p=.05$ will be used for all statistical tests reported in this article. An analysis of variance revealed that the main effect of stimulus distribution (unilateral = $477 \mathrm{msec}$; bilateral $=447 \mathrm{msec}$ ) was significant $[F(1,21)$ $=114.8, \mathrm{MSe}=172.5]$. This result essentially confirms the report by Davis and Schmit (1973) that letter pairs divided between the two sides of the visual field elicit faster responses than those presented to the same side of the visual field. The main effect of match type (physical $=437 \mathrm{msec}$; name $=487 \mathrm{msec}$ ) was also significant $[\mathrm{F}(1,21)=89.7, \mathrm{MSe}=614.6]$.

The occurrence of a significant interaction between stimulus distribution and match type $[\mathrm{F}(1,21)=53.4$, MSe $=176.2]$ was particularly interesting, in view of the 


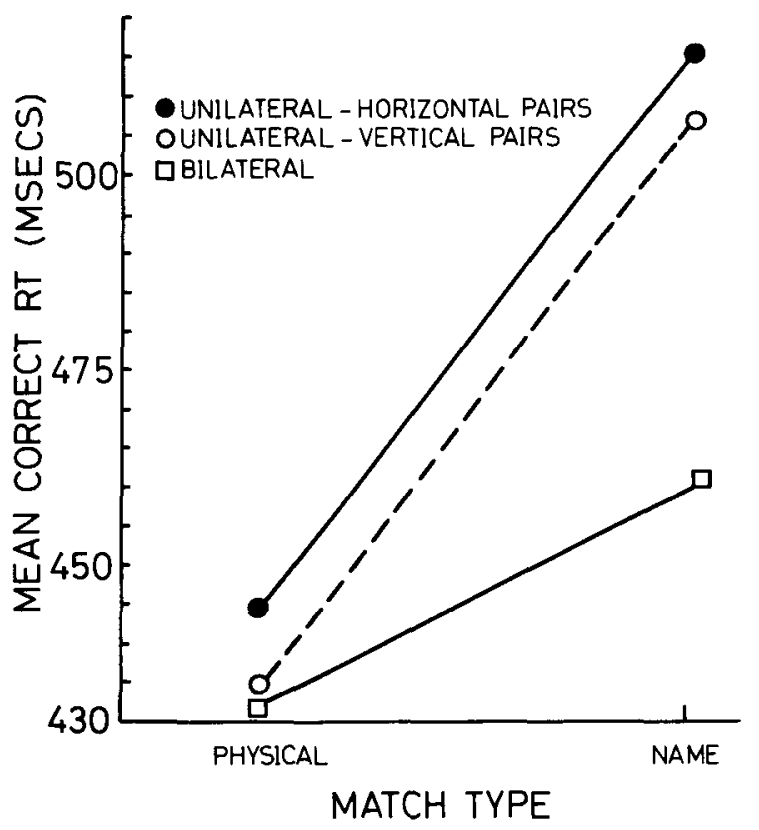

Figure 1. Mean correct RT (msec) in Experiment 1 for physical and name matches as a function of stimulus distribution and pair alignment.

fact that a specific aim of the present experiment had been to investigate the possibility of differential effects of bilateral distribution upon physical and name matches. Two planned comparisons were carried out, using a related-sample $t$ test, on the means for unilateral and bilateral trials under each type of match. It was found that the 50 -msec advantage exhibited by bilateral trials in the context of name matches was significant $[\mathrm{t}(21)=9.6]$, as was the $10-\mathrm{msec}$ advantage for bilateral presentation in the context of physical matches $[t(21)=4]$. The occurrence of a significant interaction in this study between physical and name match facilitation supports the notion that Davis and Schmit's failure to observe such an interaction may be attributed to the statistical insensitivity of their design in relation to these factors.

An analysis of variance carried out on unilateral trials alone revealed significant main effects for match type $[\mathrm{F}(1,21)=100.0, \mathrm{MSe}=2198.7]$ and pair alignment $[F(1,21)=5.1, \mathrm{MSe}=717.9]$. Interestingly, the effect of pair alignment took the form of a 9-msec advantage for vertically aligned letter pairs. This influence on performance is opposite in direction to that required to account for the effect of bilateral distribution in Davis and Schmit's study. The main effect of visual field was not significant $[F(1,21)=0.2, \mathrm{MSe}=1368.8]$, and there were no significant interactions. The absence of visual field differences suggests that the task was, as hoped, sufficiently general to obviate hemispheric specialization as a complicating factor in this investigation.

Errors. An analysis of variance carried out on error rates revealed that although the main effect of match type was significant $[\mathrm{F}(2,42)=12.8, \mathrm{MSe}=11.5]$, there was no significant effect of stimulus distribution $[F(1,21)$
$=0.3, \mathrm{MSe}=8.0]$ on errors. However, the analysis also revealed a significant interaction between match type and stimulus distribution $[\mathrm{F}(2,42)=11.5, \mathrm{MSe}=6.2]$. It can be seen from Table 1 that although error rates were lower in bilateral conditions than in unilateral conditions for both physical and name matches (i.e., "same" judgments), the reverse situation obtained in respect to "different" judgments.

The occurrence of an interaction between match type and stimulus distribution was unexpected, and it was not clear, at this stage of the investigation, how to interpret such an effect. If physical and name match errors are averaged together to provide results for "same" judgments as a whole, the ratio of "same" errors to "different" errors is $3.5 \%$ to $4.4 \%$ for unilateral displays and $1.8 \%$ to $7.1 \%$ for bilateral displays. At first sight, these values suggest that the bilateral distribution effect might be a consequence of a speed/accuracy tradeoff operating to favor "same"' RTs in bilateral trials. Such a strategy could not, however, have operated in Davis and Schmit's study. It was felt that the error data should not be accorded too much weight at this stage, since the error rates might be too low to form, by themselves, an entirely reliable basis for inference.

\section{Discussion}

The hypothesis that the vertical alignment of letter pairs retards responses to unilateral displays was clearly refuted. Instead, a small advantage in performance was found to be associated with such pairs. This result suggested that vertical alignment might enhance performance by reducing the effect of lateral interference between letters. Several investigators have reported that stimuli flanked on their peripheral boundary by a second stimulus may be subject to severe interference (e.g., Chastain \& Lawson, 1979; Wolford \& Hollingsworth, 1974). In the present context, this would suggest that recognition of the foveal-side member of a horizontal letter pair would be especially disrupted by the presence of a letter on the peripheral side. Since letter comparisons cannot proceed until both members have been identified, all subsequent operations would necessarily be delayed until the fovealside letter had been successfully processed. Vertically aligned letter pairs would escape this effect, since neither member of the pair would be subject to the disruptive influence of a more peripheral stimulus. Figure 1 shows that lateral interference might be held to account almost entirely for the apparent effect of bilateral distribution

Table 1

Percent Error for Stimulus Distribution and Match Type in Experiment 1

\begin{tabular}{lcccc}
\hline & \multicolumn{2}{c}{ "Same" } & & \\
\cline { 2 - 5 } & $\begin{array}{c}\text { Physical } \\
\text { Match }\end{array}$ & $\begin{array}{c}\text { Name } \\
\text { Match }\end{array}$ & "Different" & Mean \\
\hline Unilateral & 2.6 & 4.4 & 4.4 & 3.8 \\
Bilateral & 1.5 & 2.0 & 7.1 & 3.5 \\
Mean & 2.0 & 3.2 & 5.8 & \\
\hline
\end{tabular}


upon physical matches, and to have some significance for name matches. In view of the fact that interference has been shown to increase sharply as stimuli recede from the fovea (Estes \& Wolford, 1971; Matthews, 1973), it is interesting to note that Davis and Schmit displaced stimuli from fixation by $10^{\circ}$ of visual angle.

\section{EXPERIMENT 2}

Experiment 2 was designed to directly assess the contribution of lateral interference to the bilateral distribution effect. It has been demonstrated that, for foveal viewing, the effects of lateral interference (and some related phenomena, such as contour interference) are found at stimulus separations of less than $1^{\circ}$ of visual angle (e.g., Eriksen \& Eriksen, 1974). Thus, it is reasonable to assume that even in the presence of some degree of lateral displacement, two letters separated by more than $1^{\circ}$ of visual angle should exhibit minimal interference. Experiment 2 varied interstimulus distance as a means of manipulating lateral interference.

\section{Method}

Subjects. Twenty-four undergraduate psychology students, selected in accordance with the criteria outlined earlier, served as subjects in the experiment. The group consisted of 12 males and 12 females, ranging in age from 17 to 22 years.

Procedure. As in the previous experiment, four factors were manipulated as within-subject variables. Three of these factorsmatch type, stimulus distribution, and visual field--were implemented in the same fashion as before. The fourth factor, stimulus proximity, determined the amount of spatial separation between the letters in unilateral displays. Two levels of stimulus proximity were chosen: $0.2^{\circ}$ and $1.5^{\circ}$ of visual angle. The lower value was consistent with the separation employed in Experiment 1 , and the higher value was large enough to obviate mutual interference between the letters. Bilateral displays were produced by taking each type of unilateral display (right visual field and left visual field) and reflecting the innermost letter across the fixation point to the opposite visual field. This practice resulted in bilateral displays which were precisely equated to corresponding unilateral displays with respect to the distance of each letter from the fixation point.

Each session consisted of $\mathbf{4 8 0}$ data trials. The three levels of match type were assigned 160 trials each, and the two levels of stimulus distribution were assigned 240 trials each. Unilateral trials were further divided into 120 left- and 120 right-visual-field displays, and $1200.2^{\circ}$ separation displays and $1201.5^{\circ}$ separation displays. The $\mathbf{4 8 0}$ data trials in each session were divided into five blocks of 96 trials each. All conditions could be fully represented in their correct proportions within a cycle of 24 trials, and four such cycles were exhausted within each 96-trial block. In all other respects, this experiment resembled the previous one.

\section{Results and Discussion}

Figure 2 shows mean RT for all conditions in Experiment 2 except those pertaining to visual field. Analysis of variance revealed that the main effects of stimulus distribution [unilateral $=483 \mathrm{msec}$; bilateral $=453 \mathrm{msec}$ : $\mathrm{F}(1,23)=66.6, \mathrm{MSe}=314.0]$ and match type [physical match $=440$ msec; name match $=496$ msec: $F(1,23)=$ 92.0, $\mathrm{MSe}=815.5 \mathrm{~J}$ and the interaction between stimu-

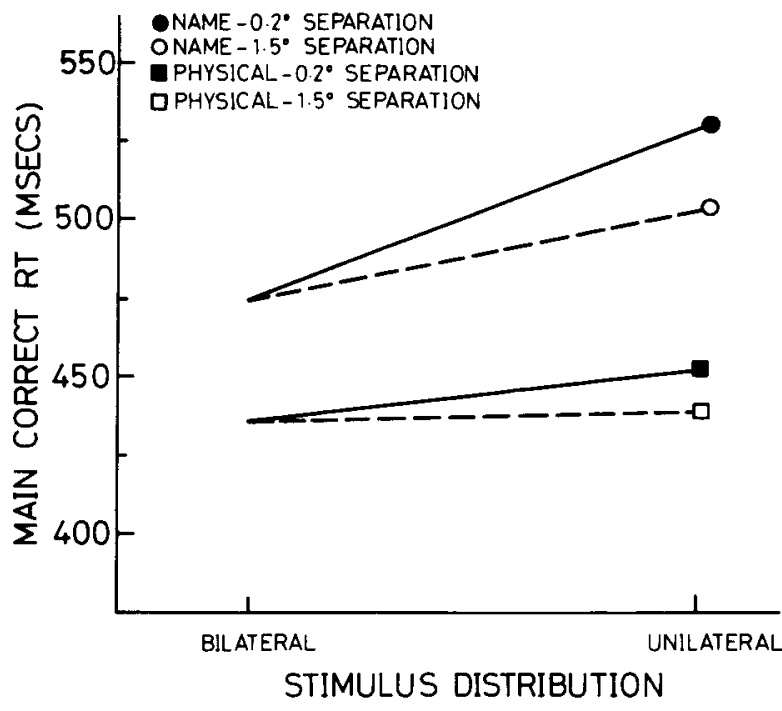

Figure 2. Mean correct RT (msec) in Experiment 2 for unilateral and bilateral trials as a function of physical and name matches at stimulus separations of 0.2 and 1.5 of visual angle.

lus distribution and match type $[\mathrm{F}(1,23)=38.9, \mathrm{MSe}=$ 112.1] were all significant.

An analysis of variance performed on unilateral conditions alone revealed significant main effects for match type $[\mathrm{F}(1,23)=95.3, \mathrm{MSe}=2427.5]$ and stimulus proximity $[F(1,23)=34.4, \mathrm{MSe}=461.2]$. The main effect of visual field was not significant $[\mathrm{F}(1,23)=1.9$, MSe $=$ 1425.0], but visual field $\times$ stimulus proximity just reached significance $[\mathrm{F}(1,23)=4.6, \mathrm{MSe}=588.3]$. No other interactions were significant in this analysis. The interaction between visual field and stimulus proximity was due to a tendency toward better performance in the left visual field than in the right when letters were separated by only $0.2^{\circ}$ of visual angle. There was a $15-\mathrm{msec}$ advantage for left visual field presentations when pair separation equaled $0.2^{\circ}$, and no advantage to either side of the visual field when the separation was extended to $1.5^{\circ}$. This finding is difficult to interpret, in view of the fact that it was absent in the previous experiment. However, it is interesting to note that such an effect is not unprecedented (e.g., Bryden, 1966; Crovitz \& Schiffman, 1965; Polich, 1978).

To evaluate the results in terms of the specific aims of the experiment, a series of individual comparisons using the Newman-Keuls procedure were carried out between the condition means represented in Figure 2:

(1) Physical matches. The Newman-Keuls tests revealed that there was a significant difference between unilateral and bilateral means at a unilateral stimulus separation of $0.2^{\circ}$ of visual angle $(\mathrm{p}<.01)$ but not at $1.5^{\circ}$ of visual angle $(\mathrm{p}>.05)$. There was, furthermore, a significant decrease in unilateral RT from $0.2^{\circ}$ to $1.5^{\circ}$ of separation $(\mathrm{p}<.01)$. These results support the view that 
the bilateral distribution effect exhibited in physical matches is the product of lateral interference between unilateral stimuli. Subjects responded significantly more slowly to unilateral displays only when the letters were separated by $0.2^{\circ}$ of visual angle.

(2) Name matches. In the context of name matches, unilateral responses were found to be significantly slower than bilateral responses at both $0.2^{\circ}(\mathrm{p}<.01)$ and $1.5^{\circ}$ ( $p<.01)$ of stimulus separation. There was, once again, a significant decrease in unilateral RT from $0.2^{\circ}$ to $1.5^{\circ}$ of separation $(p<.01)$. These findings provide further confirmation of the hypothesis generated by the results of Experiment 1 . Although separating letters by $1.5^{\circ}$ significantly reduced the difference between unilateral and bilateral conditions, there nevertheless remained a substantial effect at both levels of stimulus proximity.

Errors. An analysis of variance was conducted on the error rates shown in Table 2. The main effect of match type was found to be significant $[\mathrm{F}(2,46)=31.0, \mathrm{MSe}=$ 9.3], a result that appears to be due principally to a tendency for "different" judgments to be more susceptible to error than "same" judgments. The main effect of stimulus distribution was not significant $[F(1,23)=1.8$, $\mathrm{MSe}=7.6$, but the interaction between stimulus distribution and match type was significant $[\mathrm{F}(2,46)=6.9$, $\mathrm{MSe}=3.5]$. The overall pattern of errors represented in Table 2 is remarkably similar to that exhibited in the first experiment. When compared with unilateral error rates, bilateral errors are lower for physical and name matches ("same" judgments) and higher for "different" judgments.

This confirmation of the error pattern suggested that these effects might be better interpreted as representing a further symptom, rather than a cause, of the bilateral distribution effect. An account of the reasoning in support of this interpretation will, however, be reserved until the discussion following the next experiment, since the relevant arguments are best appreciated in the context of a more general consideration of the results of the study.

The results of Experiment 2 support the view that at least two different factors are implicated in the bilateral distribution effect. Increasing the separation between unilateral letters eliminated the effect for physical matches, but merely attenuated the effect for name matches. This evidence permits a two-component view of the bilateral distribution effect to be tentatively proposed. According to this view, lateral interference between adjacent stimuli slows responses to unilateral dis-

Table 2

Percent Error for Stimulus Distribution and Match Type in Experiment 2

\begin{tabular}{lcccc}
\multicolumn{5}{c}{ Match Type in Experiment 2 } \\
\hline & \multicolumn{2}{c}{ "Same" } & & \\
\cline { 2 - 5 } & $\begin{array}{c}\text { Physical } \\
\text { Match }\end{array}$ & $\begin{array}{c}\text { Name } \\
\text { Match }\end{array}$ & "Different" & Mean \\
\hline Unilateral & 2.6 & 3.6 & 6.0 & 4.1 \\
Bilateral & 1.4 & 1.8 & 7.1 & 3.4 \\
Mean & 2.0 & 2.7 & 6.6 & \\
\hline
\end{tabular}

plays, with a more or less equal effect upon physical and name matches. The magnitude of lateral interference in any given experimental situation will obviously depend upon such factors as stimulus separation, distance from fovea, and number, nature, and arrangement of elements (Wolford \& Shum, 1980), but it is likely that physical and name matches will be affected to a similar degree because the principal locus of interference lies in the common precategorical stage of visual encoding. Although lateral interference may account entirely for the bilateral distribution effect in physical matches, it is clear that some further factor is required to account for the major part of the effect on name matches. It is this factor which may be identified with hemispheric mechanisms.

\section{EXPERIMENT 3}

Experiment 3 was carried out to determine whether any portion of the bilateral distribution effect need be attributed to hemispheric mechanisms. It has been clearly demonstrated that the projection of stimulus material to separate hemispheres is a sufficient condition for the production of the effect, but it has not been shown that it is a necessary condition. It is conceivable, for example, that the crucial factor somehow resides in the relatively large stimulus separation inevitably associated with bilateral displays.

In view of such possibilities, studies of the bilateral distribution effect carried out to date must be considered to have seriously confounded hemispheres with stimulus separation. Experiment 3 was therefore designed as a simple, relatively unconfounded test of the importance of distributing stimuli to separate hemispheres. The strategy adopted was to separate letters in unilateral displays by virtually the same distance separating bilateral letters. It was reasoned that if nonhemispheric stimulus interaction effects of any kind were responsible for the bilateral distribution effect, then this procedure should effectively eliminate any performance difference between unilateral and bilateral conditions. If, however, hemispheric mechanisms were involved, the increased unilateral separation should be irrelevant, and the bilateral distribution effect should be unimpaired.

\section{Method}

Subjects. Twelve undergraduate psychology students, selected in the manner described earlier, served as subjects in the experiment. The sample consisted of six males and six females, ranging in age from 17 to 21 years.

Procedure. The design of Experiment 3 was relatively simple, incorporating only three within-subject variables: match type, stimulus distribution, and visual field. Physical matches were deleted in this design, leaving match type with only two levels: name match (or "same") and "different." The stimulus display utilized a geometry based on a rectangle with vertical and horizontal sides equivalent in length to $6^{\circ}$ and $5^{\circ}$ of visual angle, respectively. The fixation point was situated at the geometric center of the rectangle, and the stimuli were displayed at the corners of the rectangle. In unilateral displays, letters were centered on the top and bottom corners of the rectangle on either the right- or left-hand side. Unilateral letters were thus separated by $6^{\circ}$ of visual angle mea- 
sured from stimulus center to center. Half of all bilateral displays featured a letter at the top right corner and a letter at the bottom left corner of the rectangle; the remaining half used the top left and bottom right corners. In this case, there was a separation of $7.8^{\circ}$ of visual angle, measured diagonally from center to center. The purposes of the present experiment would have been most elegantly served by an equal amount of separation in unilateral and bilateral displays. However, this objective was not compatible with the adequate control of visual acuity differences, which demanded that bilateral letters be projected to exactly the same positions as those occupied by unilateral letters, with the exception that one letter be reflected across the vertical meridian to the corresponding position on the opposite side of the visual field. In order to obviate possible effects due to attentional scanning strategies, a simple cross equal in size to the stimulus letters always appeared in the two unoccupied corners of each display.

Four hundred and eighty data trials were presented in each session. To maintain comparability with the two previous experiments in terms of the relative frequency of "same" and "different" trials, there were 320 name match trials and 160 "different" trials. Stimulus distribution divided the session into 240 unilateral and 240 bilateral trials. Unilateral displays were further divided into 120 right- and 120 -left-visual-field trials. The 480 data trials were presented in five blocks of 96 trials each. Twelve trials sufficed to present all conditions in the correct proportions, and eight such cycles were successively exhausted in each 96-trial block. In all other respects, Experiment 3 was similar to the previous experiments.

\section{Results and Discussion}

Due to the simplicity of the experiment, three relatedsamples $t$ tests provided an adequate analysis of the RT data. The first test was performed on unilateral conditions alone and revealed no significant difference between rightand left-visual-field latencies [ $t(11)=0.8]$. The second test was carried out on bilateral conditions alone in order to evaluate the difference between the two diagonally structured displays employed in these trials. The result was just significant $[t(11)=2.9]$ : upper left/lower right displays $(546 \mathrm{msec})$ enjoyed an advantage of $10 \mathrm{msec}$ over upper right/lower left displays $(556 \mathrm{msec})$. This finding tended to confirm the suspicion that displays structured from top left to bottom right might be more compatible with normal attentional scanning patterns than those structured from top right to bottom left. A further test carried out on the difference between the means for unilateral and bilateral conditions indicated that overall bilateral responses $(551 \mathrm{msec})$ were once again significantly faster than unilateral responses $(584 \mathrm{msec})[\mathrm{t}(11)=6.6]$.

Errors. An analysis of variance was carried out on the error rates depicted in Table 3. The main effect of match type was significant $[\mathrm{F}(1,11)=22.7, \mathrm{MSe}=24.4]$, and match type $\times$ stimulus distribution was just significant $[\mathrm{F}(1,11)=6.7, \mathrm{MSe}=19.3]$, once more confirming the error pattern observed in the previous experiments. Because "same" trials occurred twice as frequently as "different" trials, the main effect of stimulus distribution was assessed by performing a $t$ test on separately computed means. The result was nonsignificant $[t(11)=0.8]$.

The results of Experiment 3 strongly suggest that mechanisms related to proximity cannot be held to account for the major part of the bilateral distribution effect. Equating stimulus separations in unilateral and bilateral
Table 3

Percent Error for Stimulus Distribution and Match Type in Experiment 3

\begin{tabular}{lccc}
\hline & "Same" & "Different" & Mean \\
\hline Unilateral & 4.1 & 7.8 & 5.3 \\
Bilateral & 1.5 & 11.6 & 4.9 \\
Mean & 2.8 & 9.7 & \\
\hline
\end{tabular}

conditions did not succeed in eliminating the usual bilateral advantage. Of course, stimulus separations in unilateral and bilateral conditions were not precisely equated, the ratio of separations being $6.0^{\circ}$ to $7.8^{\circ}$ of visual angle, respectively. However, when one compares the bilateral advantage of $32 \mathrm{msec}$ in comparable conditions of Experiment 2 , in which the separation ratio was $2.5^{\circ}$ to $8.5^{\circ}$ of visual angle, it is clear that the bilateral distribution effect is certainly not a simple function of stimulus proximity. The bilateral advantage in Experiment 3 was actually slightly larger than it was in Experiment 2, despite the fact that the separation disparity between unilateral and bilateral conditions was greatly reduced. Moreover, the unusual display geometry employed in the present experiment contributes additional support to the view that the bilateral distribution effect is not merely a product of some unidentified perceptual phenomenon peculiar to the displays most commonly used in laterality experiments.

\section{GENERAL DISCUSSION}

Taken together, Experiments 1, 2, and 3 clearly establish that letter matches are facilitated by projection of letters to separate sides of the visual field. Furthermore, these experiments demonstrate that, in the context of the GO-NOGO response procedure used in the present study, a characteristic pattern of errors is associated with this phenomenon. All three experiments showed that errors on "same" judgment trials were less frequent under bilateral presentation of stimuli, whereas errors on "different" judgment trials were less frequent under unilateral presentation of stimuli. In seeking an explanation for these data, two hemispheric models will be considered.

\section{Perceptual Analysis Model}

Under this model, originally proposed by Dimond (1972), it is assumed that stimuli projected to opposite sides of the visual field arrive in separate hemispheres of the brain and undergo simultaneous and independent perceptual processing. After perceptual processing is completed, interhemispheric integration of the resulting information is considered to occur, and further processing takes place in the normal way. On this view, the locus of the bilateral distribution effect lies in the initial operations of visual encoding. This model appears to be capable of accommodating the RT data quite comfortably. It is not difficult to accept that substantial savings in processing time might occur as a consequence of the initial operations upon stimuli being performed in separate hemi- 
spheres. The present matching paradigm, of course, introduces certain complications which must inevitably reduce overall savings. These complications relate to the necessity for interhemispheric transmission of stimulus information, the use of different hemispheric codes in comparisons, and the fact that the duration of the visual encoding process as a whole can never be less than the time required for encoding by the slower hemisphere on any given trial. Nevertheless, it seems reasonable to accept that a perceptual analysis model could account for the bilateral advantage in RT.

However, this model handles the error data less well. Bilateral presentation tended to produce fewer "same" judgment errors, but more "different" judgment scores. It is possible that some reduction in errors might occur as a consequence of the greater capacity presumed to be available for the processing of stimuli in bilateral conditions. However, although this might explain the reduction in bilateral "same" errors, it is obvious that a similar argument can be applied to "different" errors, which were, in fact, substantially greater under bilateral presentation. Furthermore, an equally compelling argument can be made for predicting relatively fewer "same" errors in unilateral conditions, based on the notion that processing two stimuli of the same name within a single system might be expected to have a facilitatory effect upon encoding that would not exist when stimuli were processed in separate systems.

A further problem for a perceptual analysis model concerns the observation that bilateral distribution differentially affected physical and name matches. Stimuli in both types of matches must undergo some form of analysis, and both should presumably benefit from hemispheric task-sharing. Some degree of bilateral facilitation should thus be evident in both physical and name match latencies. As noted earlier in this article, it is reasonable to expect physical matches to be affected less than name matches. However, the present findings indicate that, in the absence of lateral interference, bilateral distribution has no significant effect upon physical matches. A possible solution to this difficulty may lie in the observation that, even when lateral interference was controlled, physical match latencies continued to exhibit a small, though nonsignificant, advantage for bilateral stimuli. Thus, it is at least arguable that the present results simply reflect the fact that physical matches are affected by bilateral distribution to a lesser degree than are name matches, and that a more sensitive experiment would provide the required statistical confirmation for this view.

\section{Decision Process Model}

This model is based on the notion that decision processes are more likely to form a task processing bottleneck than are perceptual processes (e.g., Keele, 1973; Welford, 1968). The model assumes that the appearance of a stimulus in a particular hemifield serves to activate task processing in the contralateral hemisphere. Under unilateral presentation, only one hemisphere is activated and task processing proceeds to completion within that hemisphere. It is assumed that there is some delay before the opposite hemisphere commences task processing, if it commences task processing at all. This may be because the stimulated hemisphere is fully capable of performing the required processing and, under the speeded conditions of the experiment, is reluctant to allocate resources to the task of supporting the initiation of similar processes in the other hemisphere. Under bilateral presentation, however, the hemispheres are simultaneously stimulated and thus equally activated, and are consequently assumed to commence independent task processing at the same time. At some early stage of the task, the hemispheres will be required to exchange stimulus representations in order that comparisons may be made. This permits task processing to continue independently in each hemisphere. It is thus proposed that reduced bilateral latencies result from the operation of independent decision processes in each hemisphere. On any given trial, the response will be initiated by whichever hemisphere is first to arrive at a decision as to whether or not the stimuli match one another. On average, therefore, bilateral responses will be relatively fast, since on each trial only the response initiated by the faster hemisphere will be recognized. Under certain simplifying assumptions, this situation can be regarded as formally equivalent to the generation of an RT distribution by randomly choosing pairs of task completion times and discarding the slower member of each pair, resulting in a distribution characterized by a lower mean than the distributions from which it originates. It is apparent that the theory of order-statistics can be applied to this situation (Sarhan \& Greenberg, 1962), and in fact the requisite model is provided by the first order-statistic in a sample of size two. Under the assumption that the distribution of completion times is the same for each hemisphere, the mean and variance of the distribution of response times is given by:

$$
\begin{aligned}
& \mathrm{x}_{\mathrm{n}}=\mathrm{x}_{\mathrm{o}}-\left(\mathrm{s}^{2} / \pi\right)^{1 / 2} \\
& \mathrm{~s}_{\mathrm{n}}^{2}=\mathrm{s}_{\mathrm{o}}^{2}-\left(\mathrm{s}^{2} / \pi\right) .
\end{aligned}
$$

The reader is referred to Coney (1981) for an extended treatment of the present findings along these lines.

Figure 3 depicts the structure of the model in schematic form. This diagram is intended to show that the duplication of decision processes is the crucial feature of interest. The model does not exclude the possibility that some temporal advantage is gained under bilateral presentation from the visual encoding of stimuli in separate hemispheres, but in the interests of parsimony no such assumption has been made. The diagram also shows that no assumptions have been made as to which hemisphere (or hemispheres) is (are) involved in programming responses. This will presumably depend upon such factors as the hemisphere initiating the response, the hand used for responding, and the degree of motor control required to execute the response. The relevant difference between unilateral and bilateral presentation lies in the proposal that unilateral stimulus presentation invokes the operation of a single de- 


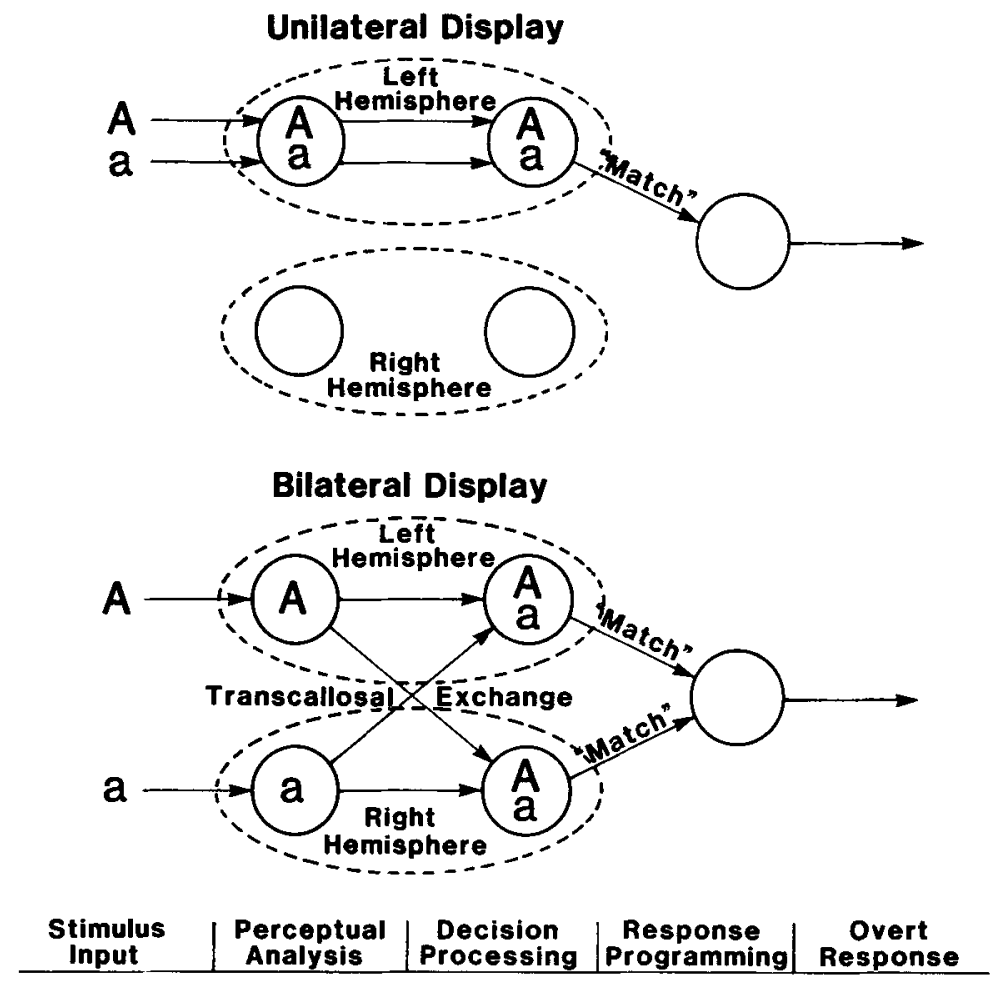

Figure 3. Schematic representation of the decision process model.

cision mechanism, whereas bilateral presentation invokes the operation of two competing decision mechanisms. The essential difference between the perceptual analysis model and the present model may be conveniently summarized by noting that the perceptual analysis model attempts to account for the bilateral distribution effect in terms of the sharing of perceptual processing, whereas the present model attributes the effect to the duplication of decision processing.

The decision process model offers an appealing explanation for the differential behavior of physical and name matches. As Expression 1 shows, the difference between unilateral and bilateral RT is a function of the variance associated with the decision process. In the present study, the variance in RT associated with physical matches is markedly less than that associated with name matches. In Experiment 1, for example, the mean standard deviation was $53 \mathrm{msec}$ for unilateral physical matches and $108 \mathrm{msec}$ for unilateral name matches. In Experiment 2, the corresponding values were 63 and $100 \mathrm{msec}$. These overall differences must reflect differences in the characteristic variance associated with the component processing stages underlying each type of match. It is reasonable to suppose, therefore, that decision times for physical matches might be less variable than those for name matches. In the context of the decision process model, this necessarily implies that physical matches would derive less advantage from bilateral distribution than would name matches. This explanation requires that physical matches exhibit at least some effect of bilateral distribution, although the expected magnitude cannot be estimated. In fact, both of the experiments that employed physical matches did show a small advantage for bilateral presentation, although the advantage was nonsignificant in Experiment 2, in which lateral interference was controlled. Further research, perhaps involving the direct manipulation of the variance associated with physical matches, is needed to settle this question.

The principal advantage of the present model derives from the way in which it is able to provide an account of the error patterns obtained in the three experiments. According to the model, unilateral error scores reflect the individual response characteristics of the decision mechanisms in each hemisphere. Let $\mathrm{Pe}+$ represent the probability of an error occurring on a unilateral "same" trial (i.e., a failure to press the response key). Then $\mathrm{Pe}+(\mathbf{r v f})$ and $\mathrm{Pe}+(\mathrm{lvf})$ represent the "same" error probabilities for the individual decision mechanisms in the left and right hemispheres, respectively. It can be shown that the use of a GO-NOGO procedure in the experiments under consideration has particular implications for bilateral error rates. Since bilateral presentation is assumed to invoke two simultaneous and independent decision processes, either hemisphere may initiate the appropriate key-closure response on the occurrence of a "same" trial. Under the GO-NOGO procedure, the appropriate "response" to "different" trials is to withhold a key closure. Because of this, a correct "same" trial response cannot be pre- 
empted by the prior occurrence of an incorrect response. Thus, an error can occur in a bilateral "same" trial only if both hemispheres fail to respond correctly. The expected probability of bilateral "same" judgment errors is therefore given by the product of the two unilateral probabilities:

$$
\mathrm{Pe}+(\text { bilateral })=\mathrm{Pe}+(\mathrm{rvf}) \cdot \mathrm{Pe}+(\mathrm{lvf}) .
$$

According to this reasoning, bilateral "same" judgment errors should always be substantially lower than unilateral "same" judgment errors. Reference to the trend in "same" judgment errors over the three experiments quickly confirms that this was the case.

Turning now to "different" judgment errors, let $\mathrm{Pe}-$ represent the probability of an error occurring on a unilateral "different" trial (i.e., a failure to withold the key closure). Then, $\mathrm{Pe}-$ (rvf) and $\mathrm{Pe}-$ (lvf) represent the "different" error probabilities for the individual decision mechanisms in the left and right hemispheres, respectively. The effect of the GO-NOGO procedure in this case is that both hemispheres are required to refrain from initiating a key closure before a correct "response" to a "different" trial can be recorded. If either hemisphere mistakenly initiates a key closure, the result is a "different" judgment error. The expected probability of bilateral "different" judgment errors is therefore given by the sum of the two unilateral probabilities:

$$
\begin{aligned}
\mathrm{Pe}-(\text { bilateral })= & \mathrm{Pe}-(\text { rvf })+\mathrm{Pe}-(\mathrm{lvf}) \\
& -\mathrm{Pe}-(\text { rvf }) \cdot \mathrm{Pe}-(\mathrm{lvf}) .
\end{aligned}
$$

Bilateral "different" judgment errors should, according to this formulation, always be substantially higher than unilateral "different" judgment errors. The "different" judgment error trends observed in all three experiments bear out this expectation.

In Table 4, Expressions 3 and 4 have been applied to unilateral error scores obtained in Experiments 1, 2, and 3 to calculate the actual bilateral error rates expected in each experiment. Observed and expected bilateral error rates are presented for comparison. In each case, it may be seen that expected "same" errors are less than observed "same" errors, and expected "different" errors are greater than observed "different" errors. The con-

Table 4

\begin{tabular}{|c|c|c|c|c|c|c|}
\hline & \multicolumn{2}{|c|}{ Experiment 1} & \multicolumn{2}{|c|}{ Experiment 2} & \multicolumn{2}{|c|}{ Experiment 3} \\
\hline & Obs & Exp & Obs & $\operatorname{Exp}$ & Obs & $\operatorname{Exp}$ \\
\hline $\begin{array}{l}\text { "Same" } \\
\text { "Different"" }\end{array}$ & $\begin{array}{l}2.0 \\
7.1\end{array}$ & $\begin{array}{l}0.2 \\
8.7\end{array}$ & $\begin{array}{l}1.8 \\
7.1\end{array}$ & $\begin{array}{r}0.1 \\
11.4\end{array}$ & $\begin{array}{r}1.5 \\
11.6\end{array}$ & $\begin{array}{r}0.2 \\
15.0\end{array}$ \\
\hline
\end{tabular}

Observed and Expected Percent Error for "Same" and "Different" Judgments on Bilateral Displays

Note-Expected values were calculated from unilateral error data, excluding data from stimulus pairs separated by $0.2^{\circ}$ of visual angle in Experiment 2. "Same" errors relate to name matches only. sistency of this discrepancy between observed and expected errors suggests that it is attributable to factors extraneous to the decision process, which are not taken into account in the present formulation. It is evident, nonetheless, that a decision process model can account for the error trends observed in Experiments 1, 2, and 3. ${ }^{1}$

Postulating mechanisms of the above kind inevitably requires the implicit acceptance of a number of assumptions, many of which may be difficult or impossible to test. Perhaps the most important of these concerns the assumption that the error probabilities associated with decisions based upon bilateral input can be predicted from the probabilities associated with input of both stimuli to one hemisphere. As noted earlier in this paper, there is evidence to suggest that each hemisphere has idiosyncratic ways of encoding similar stimulus information. It is therefore questionable whether decision processes based on information encoded either solely in the left or solely in the right hemisphere can be compared with decision processes based on stimuli encoded in separate hemispheres. This is perhaps not a major difficulty in the context of the simple, familiar stimuli employed in the present study, but may present a problem for more complex stimuli.

\section{Hemispheric Activation}

The decision process model is based on the idea that unilateral stimulation invokes only one decision process, whereas bilateral stimulation invokes two separate decision processes-one in each hemisphere. In view of the fact that the hemispheres are so richly interconnected in neural terms, it is not easy to understand why a difference between unilateral and bilateral stimulation should be sustained so far into the processing sequence. Two possibilities present themselves for consideration in this regard. The first possibility is that separate processing operations are, in fact, initiated in each hemisphere under both unilateral and bilateral stimulation, but that there is a marked asynchrony between the operations in each hemisphere in the case of unilateral stimulation. In view of the fact that in unilateral trials the nonstimulated hemisphere would be unable to commence processing until alerted by the stimulated hemisphere and provided with some representation of the stimuli, it is entirely conceivable that any decision process engendered in the nonstimulated hemisphere might be delayed beyond the point at which an effective contribution to the overt response could be made. A second possibility relates to the theory of hemispheric attentional balance developed by Kinsbourne $(1970,1975)$. Kinsbourne contends that the hemispheres are in a state of mutual inhibitory balance, and that readiness to respond to stimuli appearing on either side of the visual field is determined by the relative levels of activation of the two hemispheres. Although the empirical basis of Kinsbourne's theory has been attacked (e.g., Boles, 1979; Gardner \& Branski, 1976; Geffen, Bradshaw, \& Nettleton, 1972), his ideas have nevertheless been influential. A number of investigators have taken 
up the problem of the nature of the attentional balance between the hemispheres. For example, Heilman and Van Den Abell (1979) have shown that a warning signal directed either to the right or left side of the visual field can effect a significant reduction in simple reaction time to a stimulus subsequently presented to the same side of the visual field. They interpreted this result as evidence for the capacity of the hemispheres to be separately activated, and to sustain different levels of activation over a short period of time. Bowers and Heilman (1980) extended these findings in the context of a study involving the use of verbal and nonverbal warning signals. They found further support for the notion of sustained activational asymmetries between the hemispheres. Finally, in a series of experiments examining the effect of concurrent memory loads upon visual laterality patterns, Hellige, Cox, and Litvac (1979) have concluded that the hemispheres function as information processing systems that can be influenced somewhat separately from one another and can sustain different levels of activation. On the basis of these findings, it may be suggested that in the present paradigm the appearance of stimuli on one side or the other of the visual field serves to activate the contralateral hemisphere. Under bilateral stimulus presentation, both hemispheres will be equally and simultaneously activated. Task processing will thus proceed in parallel in both hemispheres, and overt responses will reflect the combined operation of the two processing systems. Unilateral stimulus presentation, however, will activate only one hemisphere. The opposite hemisphere will remain at a relatively lower level of activation, and may even be further depressed by inhibitory signals from its partner (Kinsbourne, 1970; Moscovitch, 1973; see, in particular, Allen's, 1983, discussion of negative interaction models of hemispheric function). Thus, task processing is likely to be substantially slower in the nonactivated hemisphere, if it occurs at all. The difference in activation levels, together with the fact that initially only the activated hemisphere has access to stimulus information, could result in an overwhelming advantage for one hemisphere under unilateral stimulus presentation. In such circumstances, it is easy to see how overt responses might be dominated by a single hemisphere under unilateral stimulation.

\section{Conclusion}

The general concept of the hemispheres as partly independent information processing systems appears to be gaining wide acceptance in recent theory and research. Most applications of this concept have been aimed principally at the relationship between independent processing and hemispheric specialization of function. The present study, however, has addressed itself directly to the problem of whether the cerebral hemispheres are capable of processing information simultaneously and independently, irrespective of their particular specialist abilities or preferences. The cumulative findings from the three experiments reported in this paper support two major conclusions. First, the argument that the bilateral distribution effect is related to hemispheric mechanisms has been supported by the results of the present investigation. Several alternative explanations have been examined and rejected, and evidence has been obtained which quite directly implicates the hemispheres in the effect. Second, the present findings not only support the idea that the hemispheres are capable of functioning as partly separate information processing systems but, in assigning the locus of the effect to decision processing, suggest that these capabilities extend a good deal further into the information processing sequence than indicated by previous research.

\section{REFERENCES}

Allen, M. (1983). Models of hemispheric specialization. Psycholog ical Bulletin, 93, 73-104.

BoLEs, D. B. (1979). Laterally biased attention with concurrent verbal load: Multiple failures to replicate. Neuropsychologia, 17, 353-361.

Bowers, D., \& HeIlman, K. M. (1980). Material-specific hemispheric activation. Neuropsychologia, 18, 309-319.

Bradshaw, J. L., \& NetTleton, N. C. (1983). Human cerebral asymmetry. Englewood Cliffs, NJ: Prentice-Hall.

BRYDEN, M. P. (1966). Left-right differences in tachistoscopic recognition: Directional scanning or cerebral dominance? Perceptual \& Motor Skills, 23, 1127-1134.

Chastain, G., \& Lawson, L. (1979). Identification asymmetry of parafoveal stimulus pairs. Perception \& Psychophysics, 26, 363-368.

Colbourn, C. J. (1978). Can laterality be measured? Neuropsychologia, 16, 283-289.

CONEY, J. R. (1981). Hemispheric processes and the bilateral distribution of stimuli in a matching task. Unpublished doctoral dissertation, University of Western Australia.

Crovitz, H. F., \& Schiffman, H. R. (1965). Visual field and the letter span. Journal of Experimental Psychology, 70, 218-223.

Davis, R., \& Schmit, V. (1973). Visual and verbal coding in the interhemispheric transfer of information. Acta Psychologica, 37, 229-240.

Dimond, S. J. (1970). Hemispheric refractoriness and control of reaction time. Quarterly Journal of Experimental Psychology, 22, 610-617.

Dimond, S. J. (1972). The double brain. Edinburgh: Churchill Livingstone.

Elithorn, A., \& Barnett, T. J. (1967). Apparent individual differences in channel capacity. Acta Psychologica, 27, 75-83.

ERIKSEN, B. A., \& ERIKSEN, C. W. (1974). Effects of noise letters upon the identification of a target letter in a nonsearch task. Perception \& Psychophysics, 16, 143-149.

ESTES, W. K., \& WOLFORD, G. L. (1971). Effects of spaces on report from tachistoscopically presented letter strings. Psychonomic Science, 25, 77-79.

Gardner, E. B., \& Branski, D. M. (1976). Unilateral cerebral activation and perception of gaps: A signal detection analysis. Neuropsychologia, 14, 43-54.

GazZaniga, M. S., \& Ledoux, J. E. (1978). The integrated mind. New York: Plenum Press.

Geffen, G., Bradshaw, J. L., \& Nettleton, N. C. (1972). Hemispheric asymmetry: Verbal and spatial encoding of visual stimuli. Joumal of Experimental Psychology, 95, 25-31.

Heilman, K. M., \& Van Den Abell, T. (1979). Right hemispheric dominance for mediating cerebral activation. Neuropsychologia, 17, 315-321.

Hellige, J. B., Cox, P. J., \& Litvac, L. (1979). Information processing in the cerebral hemispheres: Selective hemispheric activation and, capacity limitations. Journal of Experimental Psychology: General, $108,251-279$.

Heron, W. (1957). Perception as a function of retinal locus and attention. American Journal of Psychology, 70, 38-48.

KeEle, S. W. (1973). Attention and human performance. Pacific Palisades, CA: Goodyear.

Kinsbourne, M. (1970). The cerebral basis of lateral asymmetries in attention. Acta Psychologica, 33, 193-201. 
KinsBourne, M. (1975). The mechanism of hemispheric control of the lateral gradient of attention. In R. Rabbitt \& S. Dornic (Eds.), Attention and performance (Vol. V). New York: Academic Press.

KinsBourne, M. (1978). Biological determinants of functional bisymmetry and asymmetry. In M. Kinsbourne (Ed.), Asymmetrical function of the brain. Cambridge: Cambridge University Press.

MatTHEws, M. L. (1973). Locus of presentation and the selective masking effect. Canadian Journal of Psychology, 27, 343-349.

Moscovitch, M. (1973). Language and the cerebral hemispheres: Reaction-time studies and their implications for models of cerebral dominance. In P. Pliner, T. Alloway, \& L. Krames (Eds.) Communication and affect: Language and thought. New York: Academic Press.

Moscovitch, M. (1979). Information processing and the cerebral hemispheres. In M. S. Gazzaniga (Ed.), The handbook of behavioural biology: Volume on neuropsychology. New York: Plenum Press.

Pachella, R. G. (1974). The interpretation of reaction time in information-processing research. In B. Kantowitz (Ed.), Tutorials in performance and cognition. Hillsdale, NJ: Erlbaum.

Polich, J. M. (1978). Hemispheric differences in stimulus identification. Perception \& Psychophysics, 24, 49-57.

PosNer, M. I. (1970). Abstraction and the process of recognition. In J. T. Spence \& G. H. Bower (Eds.), The psychology of learning and motivation (Vol. 3). New York: Academic Press.

Posner, M. I., Boies, S. J., Eichelman, W. H., \& Taylor, R. L. (1969). Retention of visual and name codes of single letters. Journal of Experimental Psychology, 79, 1-16.

Posiner, M. I., \& MitchelL, R. F. (1967). Chronometric analysis of classification. Psychological Review, 74, 392-409.

Sarhan, A. E., \& Greenberg, B. G. (1962). Contributions to order statistics. New York: Wiley.

Swanson, J., Ledlow, A., \& KinsbourNe, M. (1978). Lateral asymmetries revealed by simple reaction time. In M. Kinsbourne (Ed.), Asymmetrical function of the brain. Cambridge: Cambridge University Press.
Welford, A. T. (1968). Fundamentals of skill. London: Methuen. WhITE, M. J. (1969). Laterality differences in perception: A review. Psychological Bulletin, 72, 387-405.

WhITE, M. J. (1973). Does cerebral dominance offer a sufficient explanation for laterality differences in tachistoscopic recognition? Perceptual \& Motor Skills, 36, 479-485.

WINER, B. J. (1971). Statistical principles in experimental design. New York: McGraw-Hill.

WOLFORD, G., \& HoLLINGSWORTH, S. (1974). Lateral masking in visual information processing. Perception \& Psychophysics, 16, 315-320.

Wolford, G., \& SHUM, K. K. (1980). Evidence for feature perturbations. Perception \& Psychophysics, 27, 409-420.

\section{NOTE}

1. All experiments in the present study employed a GO-NOGO task. It is clear from a consideration of the decision process model, however, that somewhat different predictions apply to the GO-GO or two-choice situation. Specifically, although a bilateral advantage in RT is still predicted, the error data should no longer exhibit an interaction between "same" and "different" judgments and stimulus distribution. This is because, in a GO-GO task, the action of two independent decision mechanisms cannot be distinguished from the action of a single decision mechanism. Several experiments, some of which are currently being prepared for publication, have been carried out to test these and other predictions. The results have been unequivocal. As predicted, a significant bilateral advantage in RT was observed for both "same" and "different" judgments, while the interaction between "same" and "different" judgments and stimulus distribution no longer appeared in the error data. In other words, unilateral and bilateral displays have similar effects upon "same" and "different" judgment errors in a GO-GO task.

(Manuscript received June 18, 1985; revision accepted for publication October 10, 1985.) 